\title{
Preparation and Characterization of $\left(\mathbf{I}_{2}\right)_{n}$ Sensitized Nanoporous $\mathrm{TiO}_{2}$ with Enhanced Photocatalytic Activity under Visible-Light Irradiation
}

\author{
Juzheng Zhang, ${ }^{1}$ Xin Liu, ${ }^{1}$ Shanmin Gao, ${ }^{1,2}$ Quanwen Liu, ${ }^{1}$ Baibiao Huang, ${ }^{2}$ and Ying Dai ${ }^{2}$ \\ ${ }^{1}$ School of Chemistry and Materials Science, Ludong University, Yantai 264025, China \\ ${ }^{2}$ State key labs of Crystal Materials, Shandong University, Jinan 250100, China
}

Correspondence should be addressed to Shanmin Gao, gaosm@ustc.edu and Baibiao Huang, bbhuang@sdu.edu.cn

Received 18 December 2011; Accepted 31 January 2012

Academic Editor: Xuxu Wang

Copyright () 2012 Juzheng Zhang et al. This is an open access article distributed under the Creative Commons Attribution License, which permits unrestricted use, distribution, and reproduction in any medium, provided the original work is properly cited.

A yellow/brown powder of $\left(\mathrm{I}_{2}\right)_{n}$ sensitized nanoporous $\mathrm{TiO}_{2}$ was obtained via an hydrolysis with $\mathrm{TiCl}_{4}$ and iodine hydrosol as raw material. $\mathrm{I}_{2}$ nanoparticles in the hydrosol were used as seeds to initiate the nucleation of a precursory $\mathrm{TiO}_{2}$ shell. The hybridized jumbles were further calcinated at different temperatures. The structure, crystallinity, morphology, and other physical-chemical properties of the samples are characterized by X-ray diffraction (XRD), transmission electron microscopy (TEM), $\mathrm{N}_{2}$ adsorptiondesorption isotherms measurements, and UV-vis diffuse reflectance spectroscopy (DRS). The formation mechanism of these $\left(\mathrm{I}_{2}\right)_{n}$ sensitized nanoporous $\mathrm{TiO}_{2}$ is discussed. Methylene blue solutions were used as model wastewater to evaluate the visible light photocatalytic activity of the samples. The results indicate that iodine can exist even in high-temperature calcination for iodine being encapsulated in the nanocavities inside $\mathrm{TiO}_{2}$. The degradation of methylene blue (MB) accorded with the first-order reaction model.

\section{Introduction}

Semiconductor-catalyzed photooxidation using solar light as an energy source is one of the most efficient processes for a rapid and low-cost degradation of organic pollutants. The excellent chemical stability, nontoxicity, and photoactivity of $\mathrm{TiO}_{2}$ has attracted much attention for its potential use as a solid photocatalyst for environmental purification [1-3]. However, because of its large bandgap of $3.20 \mathrm{eV}$, only a small UV fraction of solar light, about $2-3 \%$, can be utilized to generate electron-hole pairs. Hence, it is crucial to enhance the light harvesting of $\mathrm{TiO}_{2}$ in the visible region which accounts for more than $43 \%$ of the total solar energy $[4,5]$.

In general, two methods are utilized to enhance the light harvest of $\mathrm{TiO}_{2}$ in the visible region. One method is to sensitize $\mathrm{TiO}_{2}$ with color centers that have proper energy levels for photoelectron transfer between the color centers and $\mathrm{TiO}_{2}$. A cost-effective sensitization of $\mathrm{TiO}_{2}$ is typically achieved by adding nonmetals such as $\mathrm{I}_{2}[6-8]$ and $S[9,10]$. The other way is to form a doping level through intercalation of proper atoms into anatase form. For example, by doping carbon $[11,12]$ and nitrogen [1315] atoms into the $\mathrm{TiO}_{2}$ lattice, a new, weak absorption is generated at lower energies and promising approach for inducing effective Vis-absorption.

In addition to the narrow bandgap, the enhancement of surface area of the $\mathrm{TiO}_{2}$ is another important aspect for the efficiency of the materials because the desired photocatalytic reactions occur at the interfaces of the catalyst-pollute solution $[16,17]$. Mesoporous $\mathrm{TiO}_{2}$ with high specific surface area can provide abundant active sites to adsorb reactant molecules and facilitate the accessibility of reactants to the catalysts $[18,19]$. Light efficiency can also be enhanced due to the increased specific surface area and multiple scattering [20].

As a photosensitizer, element iodine not only has the absorption ability in the visible light region but also has low toxicity and can be dissolved in many solvent. Therefore, the elemental iodine can be used as a photosensitizer and prepared visible light photocatalytic materials. Titanium(IV) 
alkoxides have been frequently studied as titanium precursors, but their high cost and the need to handle organic solvents are important drawbacks. Inorganic precursors are preferable, and $\mathrm{TiO}_{2}$ powders have been often prepared by hydrolysis of $\mathrm{TiCl}_{4}$, generally in aqueous acidic solutions [21] and in pure water $[22,23]$.

Based on the above state-of-the-art designing strategies for $\mathrm{TiO}_{2}$-based photocatalysts, this paper reports the synthesis of high surface area $\left(\mathrm{I}_{2}\right)_{n}$ sensitized nanoporous $\mathrm{TiO}_{2}$. The $\mathrm{I}_{2}$ sensitized is confined preferentially in the surface/near surface region of $\mathrm{TiO}_{2}$ structure in order to accommodate hot carriers in the surface region. The $\mathrm{I}_{2}$ nanoparticles in the $\mathrm{I}_{2}$-hydrosol serve as seeds for nucleation of small $\mathrm{TiO}_{2}$ particles formed from the hydrolysis of the $\mathrm{TiCl}_{4}$. This template-free soft synthetic strategy allows the doping and pore formation to be accomplished simultaneously during calcination, while the amount and position of doping elements can be readily controlled under mild conditions.

\section{Experimental}

2.1. Materials and Sample Preparation. All chemicals used in this study were reagent grade (Tianjin Ruijinte Chemical Reagent Corp.) without further purification. Water used was deionized and doubly distilled. The $\left(\mathrm{I}_{2}\right)_{n}$ sensitized porous $\mathrm{TiO}_{2}$ samples were synthesized via the hydrolysis of $\mathrm{TiCl}_{4}$ in iodine hydrosol without using any templates or surfactants, followed by calcination at elevated temperatures.

A typical procedure for preparing the samples is described as follows. First, $0.80 \mathrm{~g}$ iodine powders were dissolved in $40 \mathrm{~mL}$ absolute ethanol and slowly added dropwisely into $100 \mathrm{~mL}$ of distilled water at $60^{\circ} \mathrm{C}$ with continuous stirring. The $\mathrm{I}_{2}$ hydrosol was formed after stirring for $0.5 \mathrm{~h}$ and then cooled to room temperature. $8 \mathrm{~mL} \mathrm{TiCl}_{4}$ was carefully added into $40 \mathrm{~mL}$-deionized water with gentle stirring in ice-water bath to avoid a drastic hydrolysis of $\mathrm{TiCl}_{4}$ in water (designated solution A). Solution A was then added dropwise with vigorous stirring into $100 \mathrm{~mL}$ of $\mathrm{I}_{2}$ hydrosol at room temperature, then the mixture solution was heated to $40^{\circ} \mathrm{C}$ and keet for $2 \mathrm{~h}$ under closed conditions. After that, a $20 \%$ (w/w) solution of ammonia was added dropwise with vigorous stirring until $\mathrm{pH}=7$, then the mixed solution was cooled down to room temperature and aged further to precipitate growth. Finally, the precipitates were centrifuged, washed with distilled water three times, and dried in an oven at $50^{\circ} \mathrm{C}$. The precipitates obtained were calcined at different temperatures in air for $3 \mathrm{~h}$. The samples after heat treatment were denoted as IT-T, where the second $\mathrm{T}$ indicates the calcination temperatures (in ${ }^{\circ} \mathrm{C}$ ). In addition, commercial P25 ( $\sim 80 \%$ anatase and $\sim 20 \%$ rutile, Deguass) was used as a reference.

2.2. Characterization. The phases of the final products were identified using X-ray diffractometer (XRD) (Rigaku $\mathrm{D} / \mathrm{max}-2500 \mathrm{VPC}$ ) with a Ni-filtered $\mathrm{Cu} \mathrm{K} \alpha$ radiation at a scanning rate of $0.02^{\circ} \mathrm{s}^{-1}$ from $20^{\circ}$ to $70^{\circ}$. The morphology of the samples was observed with transmission electron microscope (TEM, Hitachi model H800) using an accelerating voltage of $100 \mathrm{kV}$. Ultraviolet-visible (UV-vis) diffuse reflection spectra (DRS) were recorded in the range 200$800 \mathrm{~nm}$ at room temperature (Shimadzu UV-2550 UV-vis spectrophotometer). Porous structure and BET surface area were characterized by an $\mathrm{N}_{2}$ adsorption-desorption isotherm (ASAP-2020 Micromeritics Co.). Note that the samples were degassed at $180^{\circ} \mathrm{C}$ prior to the BET measurements. The pore volume and pore diameter distribution were derived from desorption branches of the isotherms by the Barrett-JoynerHalenda $(\mathrm{BJH})$ model. The BET surface area was calculated from the linear part of the Brunauer-Emmett-Teller (BET) plot.

2.3. Adsorption and Photocatalytic Activity Measurements. The adsorption capability and visible light photocatalytic activity of the $\left(\mathrm{I}_{2}\right)_{n}$ sensitized porous $\mathrm{TiO}_{2}$ were evaluated by measuring the adsorption and decomposition rate of methylene blue (MB) solution at room temperature. For comparison, the same measurements were also performed on the commercial P25. For a typical absorption and photocatalytic experiment, a total of a $200 \mathrm{mg}$ sample was added to $150 \mathrm{~mL} \mathrm{MB}$ aqueous solution $\left(5.0 \times 10^{-4} \mathrm{~mol} / \mathrm{L}\right)$ in a custom-made quartz reactor. The $\mathrm{MB}$ concentration was monitored by the UV-vis spectroscopy during the entire experiment. The solution was irradiated by visible light supplied by a $300 \mathrm{~W}$ tungsten-arc lamp (Zhejiang Electric Co. Ltd.) with a glass filter (transparent for $\lambda>400 \mathrm{~nm}$ ). After 5 minute intervals during adsorption and visible light illumination, about $3 \mathrm{~mL}$ aliquots were taken out and centrifuged to remove the trace particles. The absorbance of the centrifuged solution was measured in the range 500$800 \mathrm{~nm}$ using a UV-vis spectrophotometer (Shimadzu UV2550).

\section{Results and Discussions}

3.1. Sample Structure. The porous nanostructures were prepared via the hydrolysis of $\mathrm{TiCl}_{4}$ in $\mathrm{I}_{2}$ hydrosol, followed by calcinated in air at elevated temperatures for $3 \mathrm{~h}$. Powder $\mathrm{X}$-ray diffraction (PXRD) is used to investigate the changes of structure and crystallite sizes of the prepared porous samples at different calcination temperatures. Figure 1 shows the XRD patterns of the samples after being calcinated at various temperatures from $200^{\circ} \mathrm{C}$ to $600^{\circ} \mathrm{C}$. The $\mathrm{XRD}$ pattern for the sample obtained at $200^{\circ} \mathrm{C}$ indicated the sample predominantly amorphous titania besides trace amounts of anatase. The diffraction peaks at $2 \theta=31.6^{\circ}, 33.5^{\circ}$ and $36.0^{\circ}$ should be the iodine (JCPDS no. 71-1370). As the calcination temperatures increase, the XRD results show the pure anatase $\mathrm{TiO}_{2}$ phase (JCPDS file no. 21-172), indicating that the crystallization of the samples is achieved. In addition, as the calcination temperature increases, the peaks assigned to anatase become sharper and more intense due to the formation of larger grains as summarized in Table 1. The average crystallite sizes of $\left(\mathrm{I}_{2}\right)_{n}$ sensitized nanoporous $\mathrm{TiO}_{2}$ are 4.1, 7.2, 9.8, and $13.2 \mathrm{~nm}$ for IT-300, IT-400, IT-500, and IT-600, respectively, which are estimated from the full width 
TABLE 1: Physicochemical properties of IT-T samples from $\mathrm{N}_{2}$ sorption analysis and XRD results. ${ }^{\mathrm{a}}$

\begin{tabular}{lcccc}
\hline Sample & $\begin{array}{c}S_{\text {BET }} \\
\left(\mathrm{m}^{2} / \mathrm{g}\right)\end{array}$ & $\begin{array}{c}\text { Pore volume } \\
\left(\mathrm{cm}^{3} / \mathrm{g}\right)\end{array}$ & $\begin{array}{c}\text { Average pore size } \\
(\mathrm{nm})\end{array}$ & $\begin{array}{c}\text { Crystal size } \\
(\mathrm{nm})\end{array}$ \\
\hline IT-200 & 508.04 & 1.287 & 9.863 \\
IT-300 & 287.10 & 0.241 & 3.634 & 4.1 \\
IT-400 & 247.64 & 0.180 & 16.350 & 7.2 \\
IT-500 & 175.02 & 0.152 & 20.071 & 9.8 \\
IT-600 & 163.71 & 0.148 & 26.851 & 13.2 \\
\hline
\end{tabular}

${ }^{a}$ BET surface areas were calculated from the linear part of the BET plots. Total pore volumes were obtained from the volume of $\mathrm{N}_{2}$ adsorbed at $P / P_{0}=0.995$ Average pore diameters were estimated using the desorption branch of the isotherm and the Battett-joyner-Halenda (BJH) formula. Crystal size was determined by XRD using Scherrer equation.

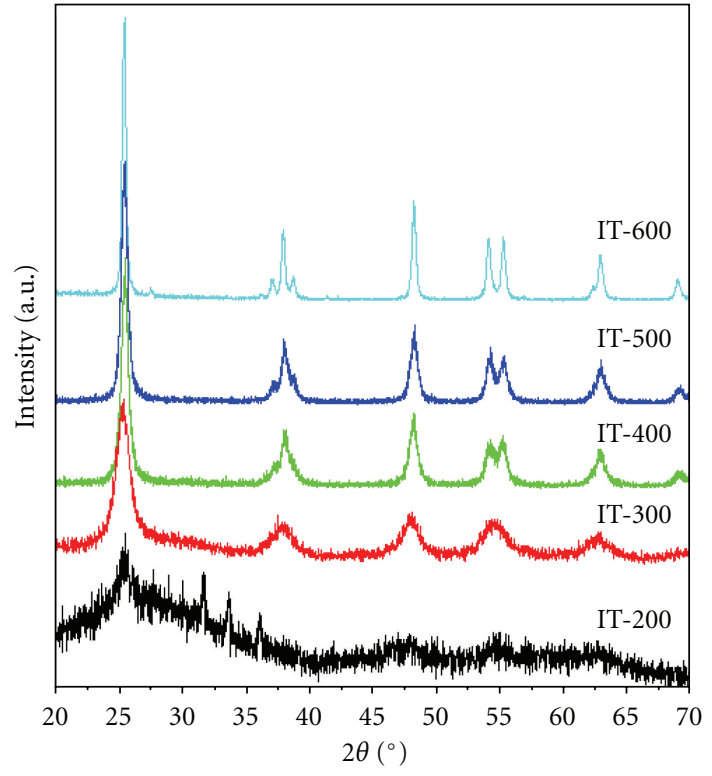

FIGURE 1: XRD patterns of the corresponding $\left(\mathrm{I}_{2}\right)_{n}$ sensitized IT-T samples after calcinated at $200^{\circ} \mathrm{C}, 300^{\circ} \mathrm{C}, 400^{\circ} \mathrm{C}, 500^{\circ} \mathrm{C}$, and $600^{\circ} \mathrm{C}$.

at half maximum of the diffraction peak using the Scherrer equation.

Figure 2 shows the TEM images of IT-T samples before and after being calcinated at different temperatures. The average particle size of the precursor is estimated to be $21 \mathrm{~nm}$, as presented in Figure 2(a). No porous structure was observed at this point. The wormhole-like irregular mesopores for $\left(\mathrm{I}_{2}\right)_{n}$ sensitized porous $\mathrm{TiO}_{2}$ can be observed in Figures 2(c)-2(f). The sample obtained at $200^{\circ} \mathrm{C}$ shows the noncrystalline nature of amorphous (Figure 2(b)), but there were porous structure. The amorphous structure is consistent with the XRD result, and the porous structure is caused by the $\mathrm{I}_{2}$ and $\mathrm{H}_{2} \mathrm{O}$ gas escape at heat treatment process. It is the subsequent heat treatment that creates the mesoporous structures. The apparent common feature of the four samples after heat treatment at $300^{\circ} \mathrm{C}, 400^{\circ} \mathrm{C}$, $500^{\circ} \mathrm{C}$ and $600^{\circ} \mathrm{C}$ is that they all consist of pseudospherical $\mathrm{TiO}_{2}$ particles. Furthermore, each individual sphere is composed of a large number of much smaller and loosely packed $\mathrm{TiO}_{2}$ nanoparticles (about 4 10 nanometers in diameter). As a result, the interstitial voids among these primary nanoparticles constitute a short-range disordered nanoporous structure. We also notice that the size of the primary nanoparticles grows with the increasing calcination temperature.

Pore size and specific surface area of the as-prepared samples were characterized by nitrogen adsorption-desorption isotherm measurements. Figure 3 shows the results obtained from $\mathrm{N}_{2}$ adsorption-desorption isotherm studies and analyzed by BET method for surface area and BJH pore size distribution. The surface area and porosity have been measured for all of the IT-T samples, and the results are given in Table 1. Calcination treatment showed a significant influence on the isotherm behavior of the samples. The VI-type isotherms for sample IT-200 and IV like Isotherm for samples IT-300, IT-400, IT-500, and IT-600 shown in Figure 3(a) demonstrate typical characteristics of mesoporous structure in the materials [24]. From the isotherms, it can be seen that the monolayer adsorption is complete when the relative pressure reaches 0.7 for sample IT-600. However, the monolayer adsorption for other samples is not complete until reaching a relative pressure of 0.8 for sample IT- 300 and 0.78 for IT-400. This indicates that sample IT-600 possesses much smaller pore size than other samples. Although mesopores contribution could be observed in Figure 3(b) for IT-600, overall pore volume measured is $0.148 \mathrm{~cm}^{3} \mathrm{~g}^{-1}$, indicating that the contribution from mesoporous is small. Less mesoporosity in IT-600 may be attributed to the large size of the particles. This result agrees with the XRD and TEM results.

Table 1 summarizes the BET-specific surface area and the apparent average pore size of our samples. The pore volume and specific surface area decrease with increasing calcination temperature. However, the samples still maintain relatively high specific surface area and pore size even for sample IT-600, which is calcined at $600^{\circ} \mathrm{C}$. Consequently, this may enhance catalytic activity, as demonstrated later in the measurement of photocatalytic activity on degradation of methylene blue.

3.2. Possible Formation Mechanism of $\left(I_{2}\right)_{n}$ Sensitized Nanoporous $\mathrm{TiO}_{2}$. Different from the conventional doping method, in which the iodine (or its precursors) is added to the matrix of $\mathrm{TiO}_{2}$, we adopt a counter strategy to add precursors 


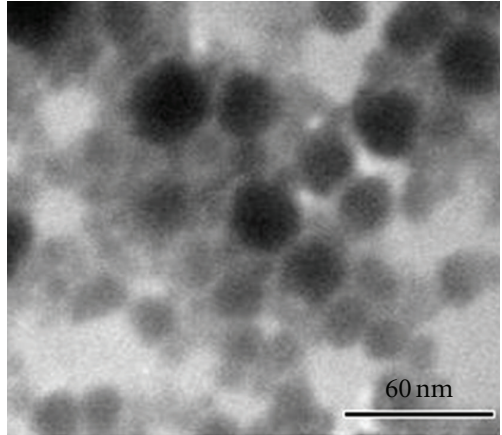

(a)

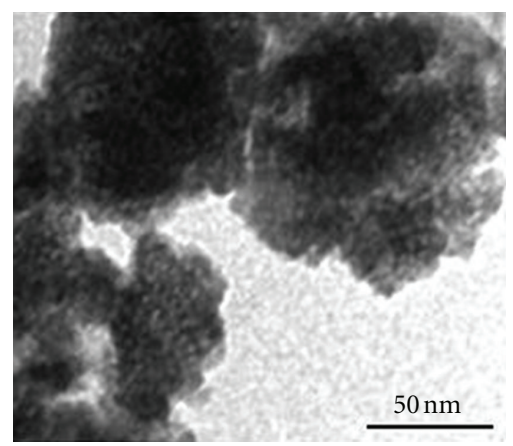

(d)

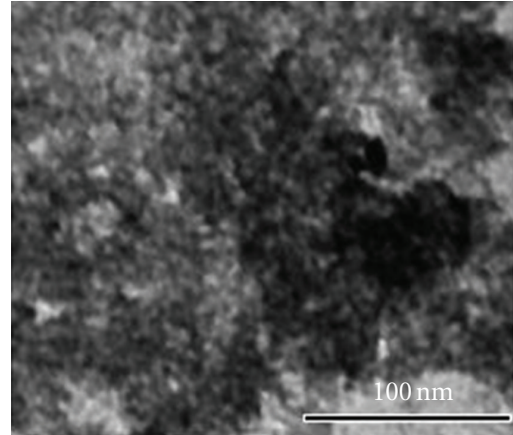

(b)

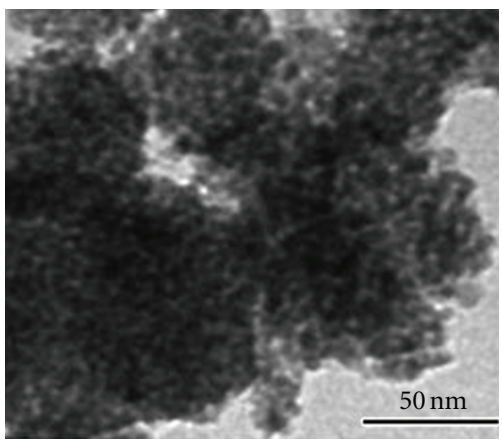

(e)

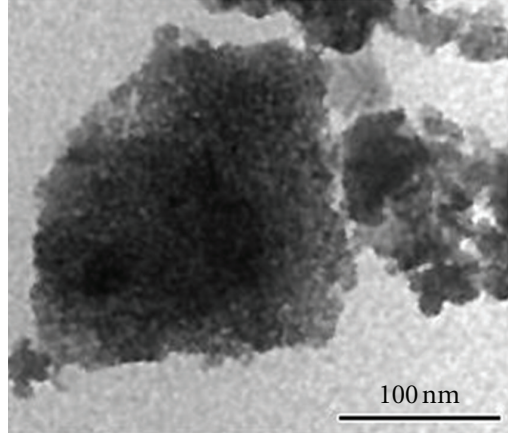

(c)

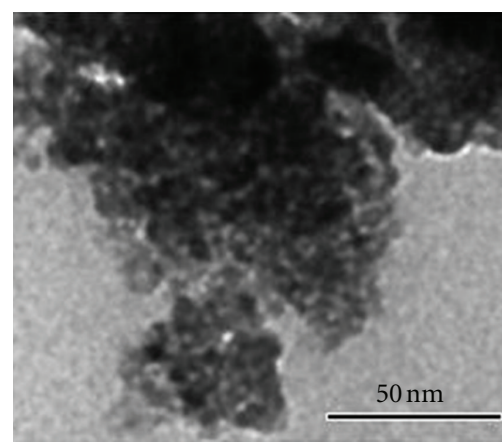

(f)

Figure 2: TEM images of the precursor of $\left(\mathrm{I}_{2}\right)_{n}$-sensitized nanoporous $\mathrm{TiO}_{2}$ (a), and IT-T calcined for $3 \mathrm{~h}$ at $(\mathrm{b}) 200^{\circ} \mathrm{C}$; $(\mathrm{c}) 300^{\circ} \mathrm{C}$; $(\mathrm{d})$ $400^{\circ} \mathrm{C}$, (e) $500^{\circ} \mathrm{C}$ and (f) $600^{\circ} \mathrm{C}$.

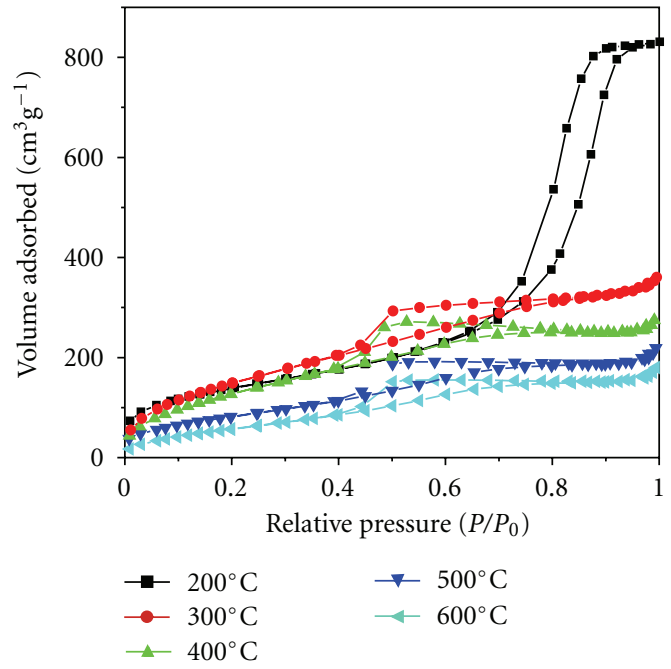

(a)

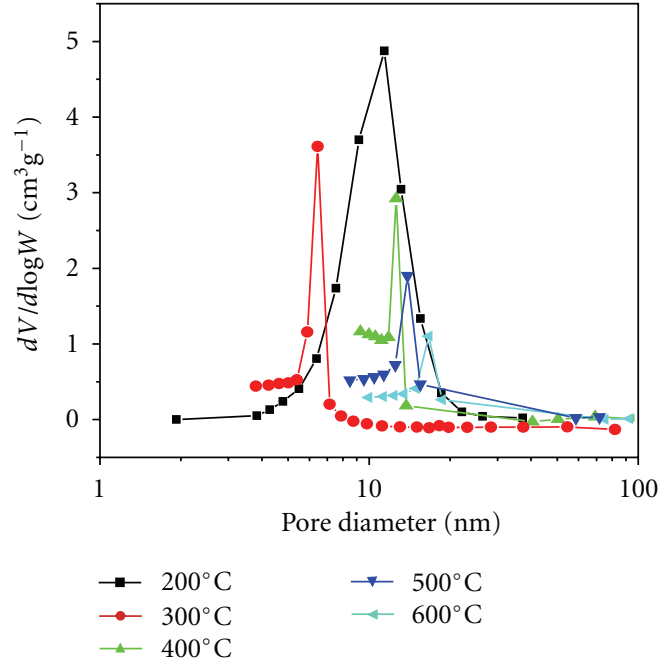

(b)

FIGURE 3: $\mathrm{N}_{2}$ adsorption/desorption isotherms (a) and the corresponding BJH pore size distributions (b) of the as-prepared IT-T samples.

of $\mathrm{TiO}_{2}$ into the matrix of $\mathrm{I}_{2}$ nanoparticles formed in the $\mathrm{I}_{2}$ hydrosol. A possible multistep mechanism, illustrated in Figure 4, is proposed to interpret the formation of the $\left(\mathrm{I}_{2}\right)_{n}$ sensitized nanoporous $\mathrm{TiO}_{2}$. When $\mathrm{TiCl}_{4}$ solution was added to $\mathrm{I}_{2}$ hydrosol, the heat of the exothermic reaction explosively generated the formation of orthotitanic acid $\left(\mathrm{Ti}(\mathrm{OH})_{4}\right)$ to become a titania precursory layer on the $\mathrm{I}_{2}$ nanoparticles [23]. These primary $\mathrm{TiO}_{2}$ precursors formed as a shell on the surface of $I_{2}$ particles. In other words, the $\mathrm{I}_{2}$ particles appear as nucleation sites for $\mathrm{TiO}_{2}$ spheres via the hydrolysis of $\mathrm{TiCl}_{4}$. At room temperature, the hydrolysis of $\mathrm{TiCl}_{4}$ is relatively slow. When the starting solution was heated in an isothermal water bath of $40^{\circ} \mathrm{C}$ for $2 \mathrm{~h}$ and add ammonia solution, it became supersaturated 


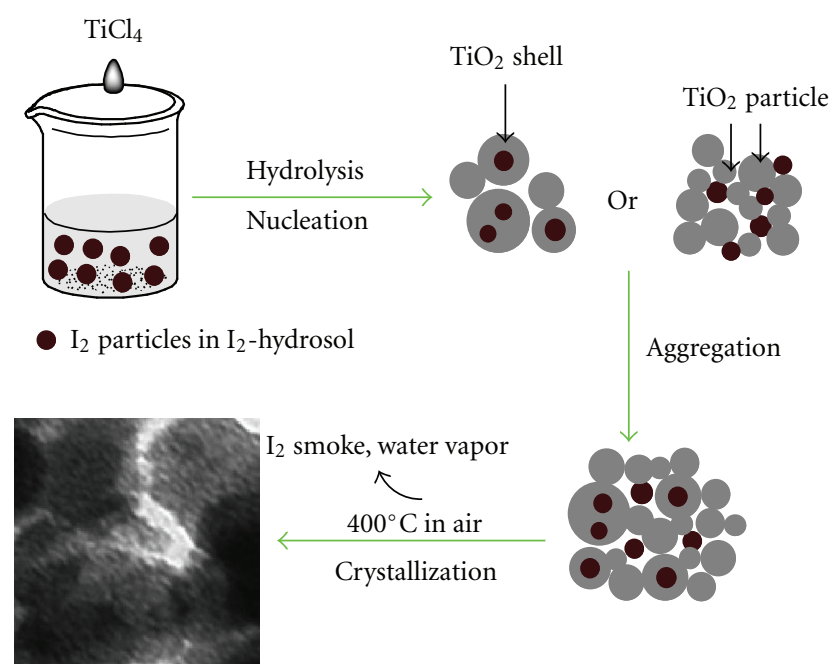

Figure 4: Proposed formation mechanism of $\left(\mathrm{I}_{2}\right)_{n}$ sensitized nanoporous $\mathrm{TiO}_{2}$.

and precipitated. After drying the precursor turn into $\left(\mathrm{I}_{2}\right)_{n^{-}}$ $\mathrm{H}_{2} \mathrm{TiO}_{3}$. The subsequent calcinated of the precipitated at elevated temperatures leading to a series of reactions among $\mathrm{I}_{2}$ and $\mathrm{TiO}_{2}$ precursors with the presence of $\mathrm{O}_{2}$. We believe that the $\left(\mathrm{I}_{2}\right)_{n}$-sensitized nanoporous $\mathrm{TiO}_{2}$ is a product of the following synergetic processes, including (1) the $I_{2}$ sublimes and the thermal decomposition $\mathrm{H}_{2} \mathrm{TiO}_{3}$ resulting gaseous products pulverize the $\mathrm{TiO}_{2}$ shell, leading to the formation of the primary pores. (2) Part of the $\mathrm{I}_{2}$ attaches to surface of the $\mathrm{TiO}_{2}$ primary particles. The further oxidation of $\mathrm{I}_{2}$ is more or less prevented by the shell of primary $\mathrm{TiO}_{2}$ nanoparticles [7]. (3) The amorphous $\mathrm{TiO}_{2}$ crystallizes into anatase $\mathrm{TiO}_{2}$.

3.3. UV-Vis Diffuse Reflectance Spectra. Figure 5 collects the UV-Vis diffuse reflectance spectra of the as-synthesized samples, the $\mathrm{P} 25$, as well as the $\mathrm{I}_{2}$ hydrosol. In comparison to the pure $\mathrm{TiO}_{2}$, the spectra of $\left(\mathrm{I}_{2}\right)_{n}$ sensitized nanoporous $\mathrm{TiO}_{2}$ samples exhibit a strong broad absorption band between 400 and $750 \mathrm{~nm}$, covering nearly the entire visible range. This band is centered around $500 \mathrm{~nm}$, which is the spectral range where iodine absorbs [7]. Apparently, the absorbance between 400 and $700 \mathrm{~nm}$ decreases for sample prepared at higher temperatures. This can be ascribed to the additional loss of $\mathrm{I}_{2}$ by sublimation at higher temperatures. But the sample which is obtained at $600^{\circ} \mathrm{C}$ still has a strong absorption in the visible region. Nonetheless, the absorption in the visible-light region implies that the prepared $\left(\mathrm{I}_{2}\right)_{n}$ sensitized nanoporous $\mathrm{TiO}_{2}$ samples can be activated by visible light, and that more photogenerated electrons and holes can be created and they can participate in the photocatalytic oxidation reactions. We believe that, with the increase of the temperature during heat treatment, the concentration of iodine molecules on the surface of the IT samples decreased due to sublimation of $I_{2}$ at high temperature, which is also

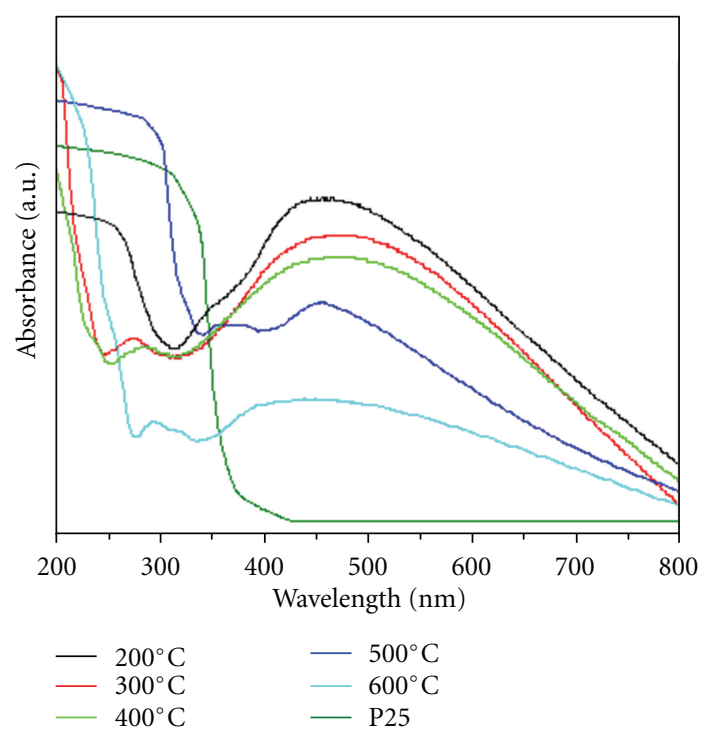

FIGURE 5: UV-vis diffuse reflectance spectra of IT-T catalysts at various calcination temperatures compared to P25.

confirmed by the color change of the samples during the heat treatment. The color of the samples changed from puce, to brown, slight brown and fawn, and finally to light gray when the temperature increased from 200 to $600^{\circ} \mathrm{C}$.

3.4. Photocatalytic Activity on the Decomposition of Methylene Blue. Methylene blue (MB) is a brightly colored blue cationic thiazine dye and is often used as a test model pollutant in semiconductor photocatalysis. A blank experiment was also carried out. The pure MB solution cannot decompose without catalyst. Figure 6(a) shows the adsorption and photodegradation ability on MB solutions of our IT-T samples in comparison to P25. Prior to the turn-on of the light, the concentrations of $\mathrm{MB}$ in the presence of the IT-T samples deplete much faster than P25. This is especially seen for IT-200 sample; nearly $70 \%$ of the dye molecules were adsorbed within $30 \mathrm{~min}$. We ascribe this effect to the much larger surface area of the IT-T samples than that of P25 as characterized and discussed above. For IT-T samples, their adsorption capacity increases with the decrease of the calcination temperatures. This can be credited to the reduced surface area.

Furthermore, Figure 6(a) shows, under visible light illumination, the MB solutions containing the IT-T samples underwent significant degradation and became nearly transparent within $50 \mathrm{~min}$. In contrast, the $\mathrm{MB}$ solutions containing P25 show very limited degradation. It should be noted that IT-400 sample showed the highest photocatalytic oxidation on methylene blue. This is because the crystalline of IT-400 is well and has fewer defects, so the recombination of electron and hole is very low. In addition, the sample still has larger surface area, so IT-400 has good absorption capacity.

The photocatalytic activity of the powders can be quantitatively evaluated by comparing the apparent reaction rate 


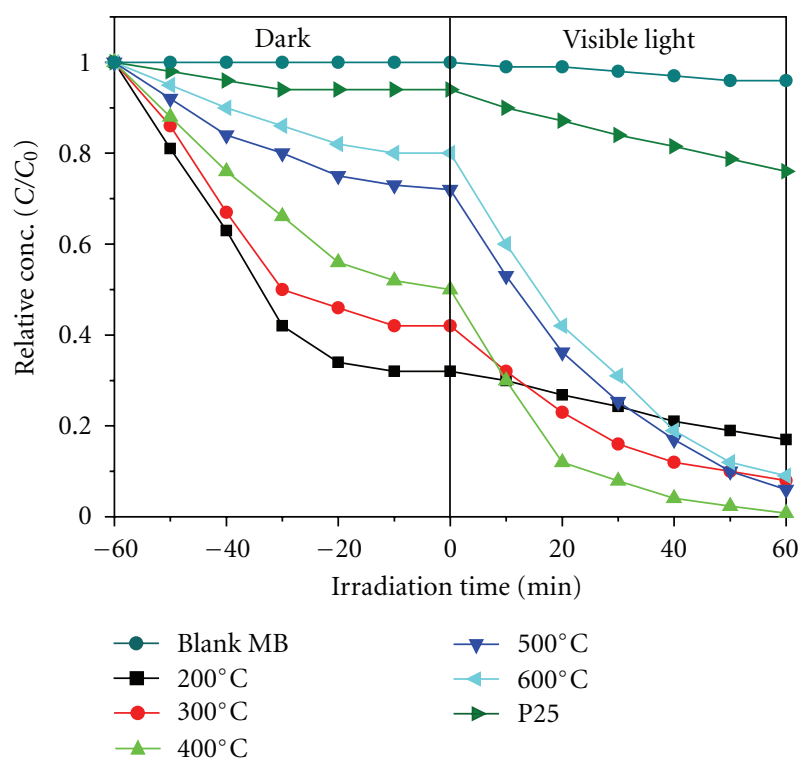

(a)

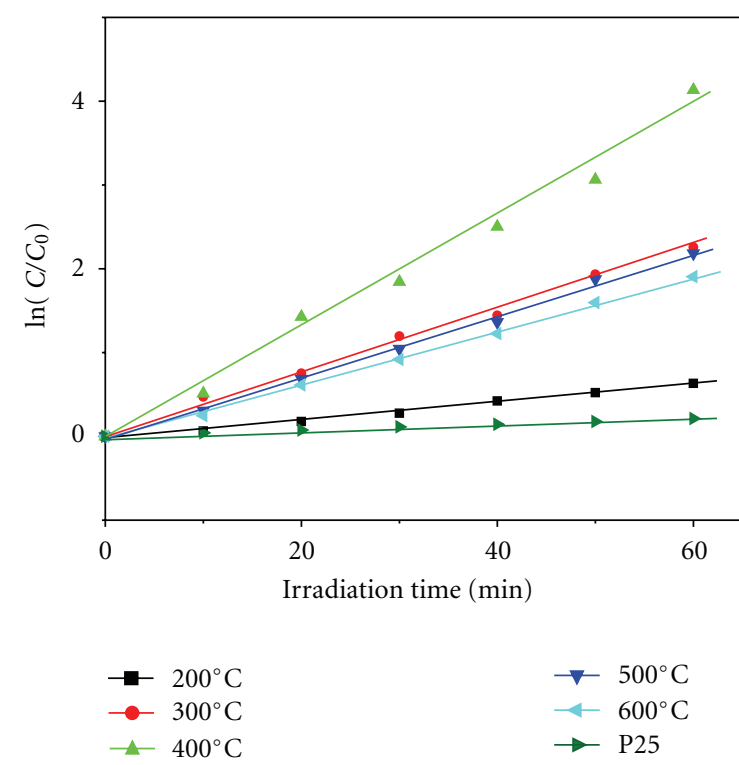

(b)

FIgURE 6: (a) The blank MB solution, photodegradation of methylene blue solutions by using IT-T and P25 under visible light in a neutral suspension. (b) The variation of normalized $\ln \left(C_{0} / C\right)$ of $\mathrm{MB}$ concentration as a function of visible light irradiation time.

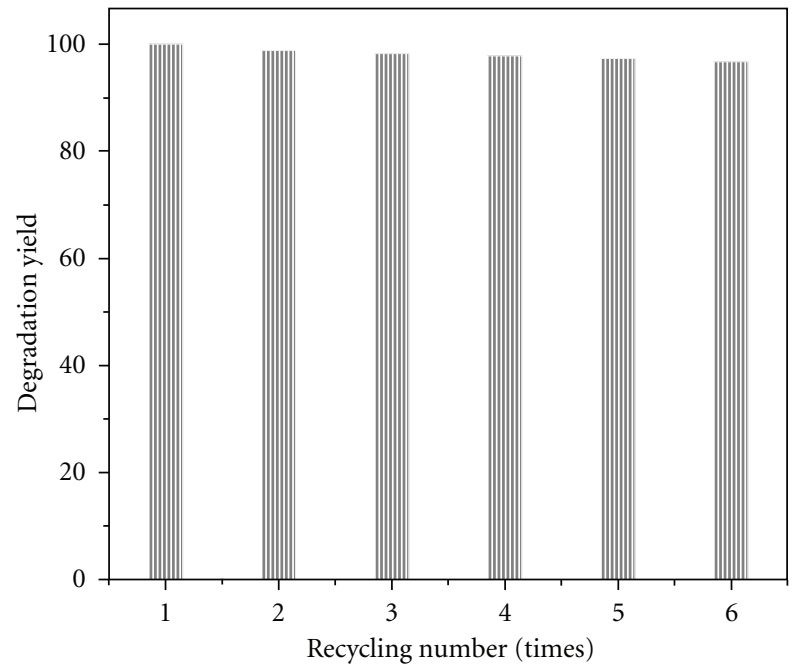

Figure 7: Recycling test result by using the IT-400 sample.

constants. It is well known that photocatalytic oxidation of organic pollutants in aqueous suspension follows the Langmuir-Hinshelwood model [25], and it can be described as follows [26]:

$$
\ln \left(\frac{C_{0}}{C_{t}}\right)=k_{\text {app }} \times t
$$

where $C_{t}$ is the concentration of $\mathrm{MB}$ aqueous at reaction time $t, C_{0}$ is the initial $\mathrm{MB}$ concentration, $k_{\text {app }}$ is apparent rate constant, and $t$ is reaction time. The variations in $\ln \left(C_{0} / \mathrm{C}\right)$ as a function of irradiation time are given in Figure 6(b). Since all the curves can be fitted roughly to a straight line, the photocatalytic degradation reaction can be assumed

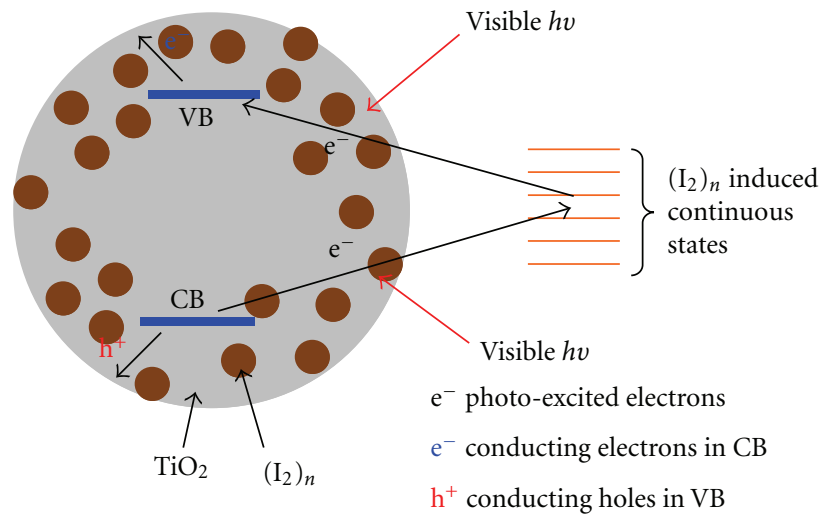

Figure 8: Proposed visible light-driven photocatalytic mechanism on the $\left(\mathrm{I}_{2}\right)_{n}$ sensitized nanoporous $\mathrm{TiO}_{2}$ nanoparticle.

to follow first-order kinetics [27]. The rate constant for the photodegradation of the MB using IT-400 is 21 times higher than that of P25. All together, we can conclude that IT-T samples exhibited a strong photocatalytic activity for decomposition of $\mathrm{MB}$ under visible light irradiation.

The cyclic stability test under the same conditions of IT-400 sample was also examined and shows excellent photocatalytic activity in degrading MB solution, as shown in Figure 7. No significant decrease in its photocatalytic oxidation activity is observed after being used 6 times, which makes it very promising for practical application.

\subsection{Proposed Mechanism for the Enhanced Photocatalytic} Activity. The enhanced photocatalytic oxidation activity in IT-T samples is a product of several factors. (1) The 
sensitized of $\left(\mathrm{I}_{2}\right)_{n}$ introduces continuous states residing in between the $\mathrm{VB}$ and $\mathrm{CB}$ of $\mathrm{TiO}_{2}$ [28]. As schematically elucidated in Figure 8, we believe these I-induced continuous states can either accept visible light-excited electrons from the $\mathrm{VB}$ of $\mathrm{TiO}_{2}$ and/or provide visible-light excited electrons from these $\mathrm{I}_{2}$-induced states to the $\mathrm{CB}$ of $\mathrm{TiO}_{2}$. (2) Due to the nature of our preparing method, the sensitized $\mathrm{I}_{2}$ must be preferentially located in the surface region of the $\mathrm{TiO}_{2}$ nanoparticles. Consequently, the I-induced defective states are preferentially concentrated in the surface region instead of the bulk of the $\mathrm{TiO}_{2}$, which facilitate the trap of hot carrier in the surface region where the desired photodegradation reactions occur $[6,29]$. (3) Because the photo-degradation occurs at the surface of $\mathrm{TiO}_{2}$, the organic pollute molecules must be preconcentrated at the $\mathrm{TiO}_{2}$ surface in order to react with the trap hot carriers and the reactive radicals. Thus, the high surface areas of the nanoporous $\mathrm{TiO}_{2}$ enhance the adsorption of methylene blue molecules onto the surface of $\mathrm{TiO}_{2}$ particles. This is evidenced by the pronounced more and faster deletion of methylene blue molecules in solution under darkness (Figure 6(a)) in comparison to P25 sample. In addition, large surface area can accommodate more surface-adsorbed water and hydroxyl groups that act as photoexcited hole traps and produce hydroxyl radicals for the degradation of organic pollute molecules [30].

\section{Conclusions}

In summary, a unique template-free synthetic strategy is used to prepare $\left(\mathrm{I}_{2}\right)_{n}$ sensitized nanoporous $\mathrm{TiO}_{2}$ through a hydrolysis-calcination process. $\mathrm{I}_{2}$ hydrosol is used as nucleation center and sensitizer, while the $\mathrm{TiCl}_{4}$ was used as the precursor for $\mathrm{TiO}_{2}$. The calcination temperature has a crucial role in the amount of $\left(\mathrm{I}_{2}\right)_{n}$ sensitized nanoporous $\mathrm{TiO}_{2}$. The calcination enhanced the phase transformation of the $\mathrm{TiO}_{2}$ powders form amorphous to anatase phases and crystallization of anatase. The photocatalytic activity of the as-prepared samples was higher than that of P25 for the degradation of $\mathrm{MB}$. The sample calcined at $400^{\circ} \mathrm{C}$ shows the highest photocatalytic activity in the decomposition of methylene blue under visible light due to the enhanced absorption in visible region and the large specific surface area.

\section{Acknowledgments}

S. Gao and B. Huang thank the National Basic Research Program of China (973 Program, no. 2007CB613302), Doctoral Foundation of Shandong Province (2007BS04040), and Project of Shandong Province Higher Educational Science and Technology Program (J10LF02).

\section{References}

[1] A. L. Linsebigler, G. Q Lu, and J. T. Yates, "Photocatalysis on $\mathrm{TiO}_{2}$ surfaces: principles, mechanisms, and selected results," Chemical Reviews, vol. 95, no. 3, pp. 735-758, 1995.

[2] J. Zhang, Q. Xu, Z. C. Feng, M. J. Li, and C. Li, "Importance of the relationship between surface phases and photocatalytic activity of $\mathrm{TiO}_{2}$," Angewandte Chemie, vol. 47, no. 9, pp. 1766$1769,2008$.

[3] H. F. Cheng, B. B. Huang, P. Wang et al., "In situ ion exchange synthesis of the novel $\mathrm{Ag} / \mathrm{AgBr} / \mathrm{BiOBr}$ hybrid with highly efficient decontamination of pollutants," Chemical Communications, vol. 47, no. 25, pp. 7054-7056, 2011.

[4] H. P. Li, W. Zhang, and W. Pan, "Enhanced photocatalytic activity of electrospun $\mathrm{TiO}_{2}$ nanofibers with optimal anatase/rutile ratio," Journal of the American Ceramic Society, vol. 94, no. 10, pp. 3184-3187, 2011.

[5] X. N. Wang, B. B. Huang, Z. Y. Wang et al., "Synthesis of Anatase $\mathrm{TiO}_{2}$ tubular structures microcrystallites with a high percentage of $\{001\}$ facets by a simple one-step hydrothermal template process," Chemistry, vol. 16, no. 24, pp. 7106-7109, 2010.

[6] P. Xu, J. Lu, T. Xu, S. M. Gao, B. B. Huang, and Y. Dai, " $\mathrm{I}_{2}$-hydrosol-seeded growth of $\left(\mathrm{I}_{2}\right)_{n}$-C- codoped meso/ nanoporous $\mathrm{TiO}_{2}$ for visible light-driven photocatalysis," Journal of Physical Chemistry C, vol. 114, no. 20, pp. 95109517, 2010.

[7] S. Usseglio, A. Damin, D. Scarano, S. Bordiga, A. Zecchina, and C. Lamberti, " $\left(\mathrm{I}_{2}\right)_{n}$ encapsulation inside $\mathrm{TiO}_{2}$ : a way to tune photoactivity in the visible region," Journal of the American Chemical Society, vol. 129, no. 10, pp. 2822-2828, 2007.

[8] P. C. Deng, H. Z. Wang, B. W. Sun, L. X. Fan, and L. Li, "Hydrothermal preparation and photocatalytic performance of $\left(\mathrm{I}_{2}\right)_{n}$ Sensitized and $\mathrm{I}(\mathrm{V})$ Doped $\mathrm{TiO}_{2}$ Supported on $\mathrm{SiO}_{2}$," Journal of Advanced Oxidation Technologies, vol. 12, no. 2, pp. 226-230, 2009.

[9] P. Xu, T. Xu, J. Lu et al., "Visible-light-driven photocatalytic $\mathrm{S}$ - and C- codoped meso/nanoporous $\mathrm{TiO}_{2}$," Energy and Environmental Science, vol. 3, no. 8, pp. 1128-1134, 2010.

[10] H. X. Li, X. Y. Zhang, Y. N. Huo, and J. Zhu, "Supercritical preparation of a highly active S-doped $\mathrm{TiO}_{2}$ photocatalyst for methylene blue mineralization," Environmental Science and Technology, vol. 41, no. 12, pp. 4410-4414, 2007.

[11] S. Sakthivel and H. Kisch, "Daylight photocatalysis by Carbonmodified Titanium Dioxide," Angewandte Chemie, vol. 42, no. 40, pp. 4908-4911, 2003.

[12] G. S. Wu, T. Nishikawa, B. Ohtani, and A. C. Chen, "Synthesis and characterization of carbon-doped $\mathrm{TiO}_{2}$ nanostructures with enhanced visible light response," Chemistry of Materials, vol. 19, no. 18, pp. 4530-4537, 2007.

[13] R. Asahi, T. Morikawa, T. Ohwaki, K. Aoki, and Y. Taga, "Visible-light photocatalysis in nitrogen-doped titanium oxides," Science, vol. 293, no. 5528, pp. 269-271, 2001.

[14] J. Wang, D. N. Tafen, J. P. Lewis et al., "Origin of photocatalytic activity of Nitrogen-doped $\mathrm{TiO}_{2}$ nanobelts," Journal of the American Chemical Society, vol. 131, no. 34, pp. 12290-12297, 2009.

[15] Q. Wang, C. C. Chen, W. H. Ma, H. Y. Zhu, and J. C. Zhao, "Pivotal role of fluorine in tuning band structure and visible-light photocatalytic activity of nitrogen-doped $\mathrm{TiO}_{2}$," Chemistry, vol. 15, no. 19, pp. 4765-4769, 2009.

[16] Z. Y. Liu, H. W. Bai, and D. Sun, "Facile fabrication of hierarchical porous $\mathrm{TiO}_{2}$ hollow microspheres with high photocatalytic activity for water purification," Applied Catalysis B, vol. 104, no. 3-4, pp. 234-238, 2011.

[17] Y. Ide, Y. Nakasato, and M. Ogawa, "Molecular recognitive photocatalysis driven by the selective adsorption on layered titanates," Journal of the American Chemical Society, vol. 132, no. 10, pp. 3601-3604, 2010.

[18] Y. Shiraishi, N. Saito, and T. Hirai, "Adsorption-driven photocatalytic activity of mesoporous titanium dioxide," Journal 
of the American Chemical Society, vol. 127, no. 37, pp. 1282012822, 2005.

[19] P. Raveendran, M. Eswaramoorthy, U. Bindu et al., "Templatefree formation of meso-structured anatase $\mathrm{TiO}_{2}$ with spherical morphology," Journal of Physical Chemistry C, vol. 112, no. 50, pp. 20007-20011, 2008.

[20] J. Fang, F. Wang, K. Qian, H. Bao, Z. Q. Jiang, and W. X. Huang, "Bifunctional N-doped mesoporous $\mathrm{TiO}_{2}$ photocatalysts," Journal of Physical Chemistry C, vol. 112, no. 46, pp. 18150-18156, 2008.

[21] C. A. Nolph, D. E. Sievers, S. Kaewgun et al., "Photocatalytic study of polymorphic titania synthesized by ambient condition sol process," Catalysis Letters, vol. 117, no. 3-4, pp. 102106, 2007.

[22] Y. Z. Li, Y. N. Fan, and Y. Chen, "A novel method for preparation of nanocrystalline rutile $\mathrm{TiO}_{2}$ powders by liquid hydrolysis of $\mathrm{TiCl}_{4}$," Journal of Materials Chemistry, vol. 12, no. 5, pp. 1387-1390, 2002.

[23] A. D. Paola, M. Bellardita, R. Ceccato, L. Palmisano, and F. Parrino, "Highly active photocatalytic $\mathrm{TiO}_{2}$ powders obtained by thermohydrolysis of $\mathrm{TiCl}_{4}$ in water," Journal of Physical Chemistry C, vol. 113, no. 34, pp. 15166-15174, 2009.

[24] K. S. W. Singh, D. H. Everett, R. A. W. Haul et al., "Reporting physisorption data for gas/solid systems," Pure and Applied Chemistry, vol. 57, no. 4, pp. 603-619, 1985.

[25] C.-H. Wu, H.-W. Chang, and J.-M. Chern, "Basic dye decomposition kinetics in a photocatalytic slurry reactor," Journal of Hazardous Materials, vol. 137, no. 1, pp. 336-343, 2006.

[26] J. G. Yu, G. H. Wang, B. Cheng, and M. H. Zhou, "Effects of hydrothermal temperature and time on the photocatalytic activity and microstructures of bimodal mesoporous $\mathrm{TiO}_{2}$ powders," Applied Catalysis B, vol. 69, no. 3-4, pp. 171-180, 2007.

[27] J. M. Wu and T. W. Zhang, "Photodegradation of rhodamine $B$ in water assisted by titania films prepared through a novel procedure," Journal of Photochemistry and Photobiology A, vol. 162, no. 1, pp. 171-177, 2004.

[28] S. Tojo, T. Tachikawa, M. Fujitsuka, and T. Majima, "Iodinedoped $\mathrm{TiO}_{2}$ photocatalysts: correlation between band structure and mechanism," Journal of Physical Chemistry C, vol. 112, no. 38, pp. 14948-14954, 2008.

[29] M. R. Hoffmann, S. T. Martin, W. Choi, and D. W. Bahnemann, "Environmental applications of semiconductor photocatalysis," Chemical Reviews, vol. 95, no. 1, pp. 69-96, 1995.

[30] V. Subramanian, E. E. Wolf, and P. V. Kamat, "Catalysis with $\mathrm{TiO}_{2}$ /gold nanocomposites. Effect of metal particle size on the fermi level equilibration," Journal of the American Chemical Society, vol. 126, no. 15, pp. 4943-4950, 2004. 


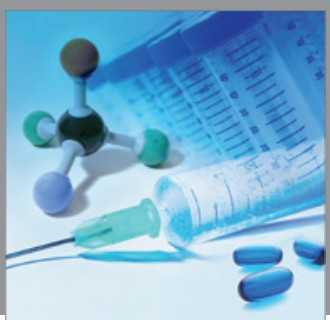

International Journal of

Medicinal Chemistry

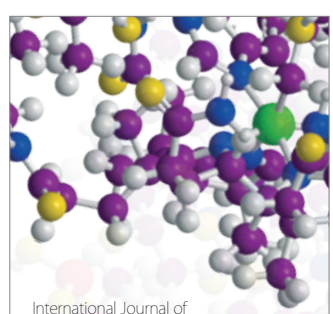

Carbohydrate Chemistry

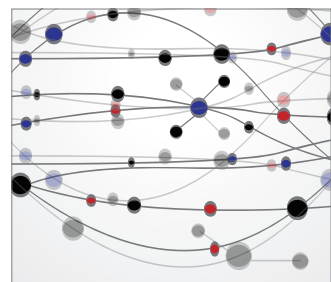

The Scientific World Journal
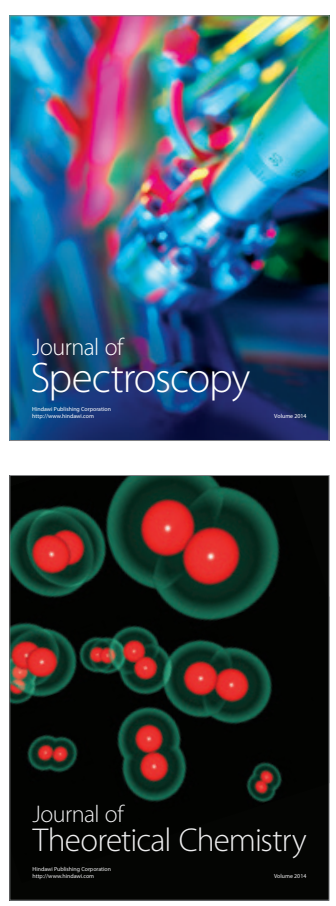
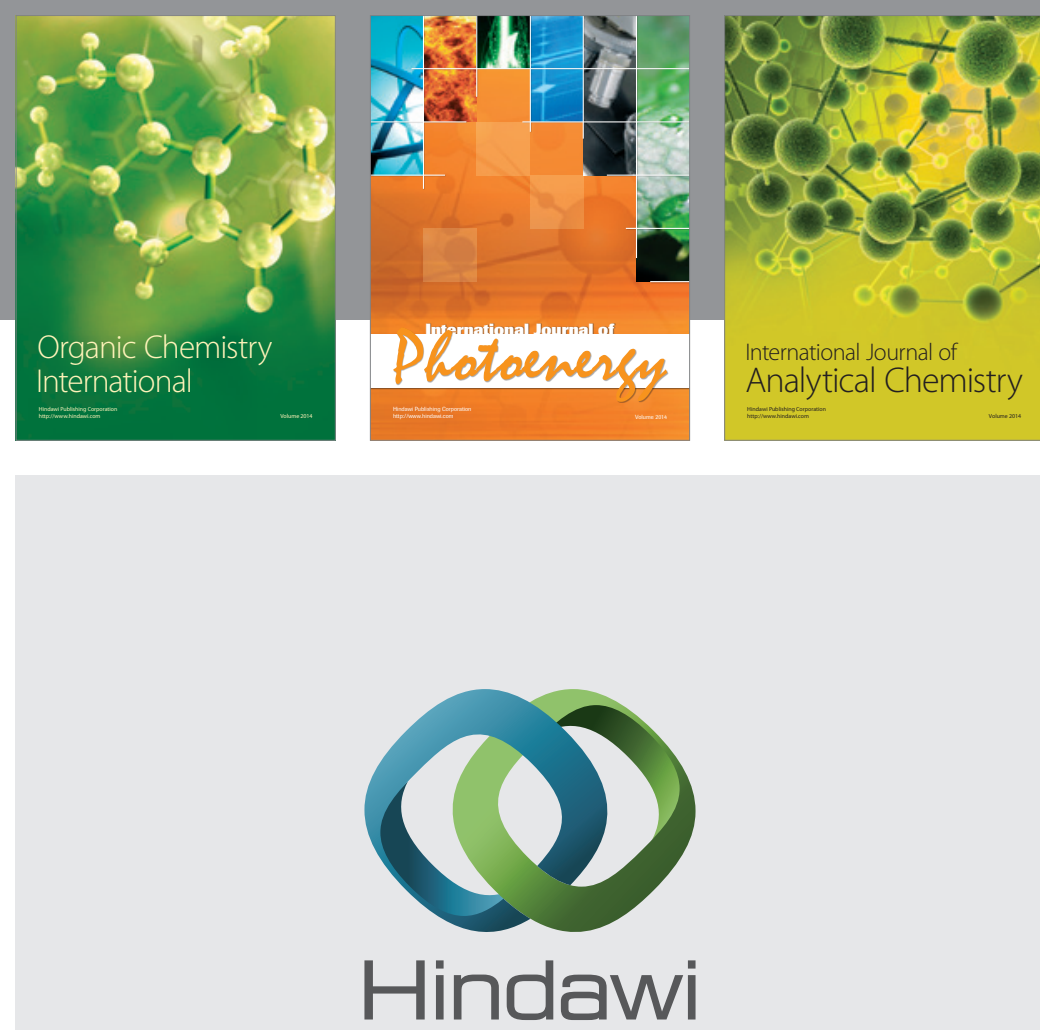

Submit your manuscripts at

http://www.hindawi.com
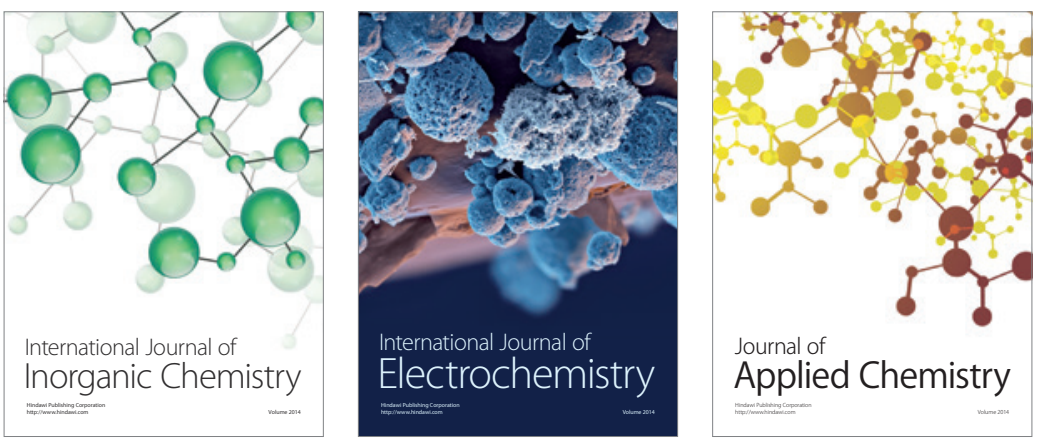

Journal of

Applied Chemistry
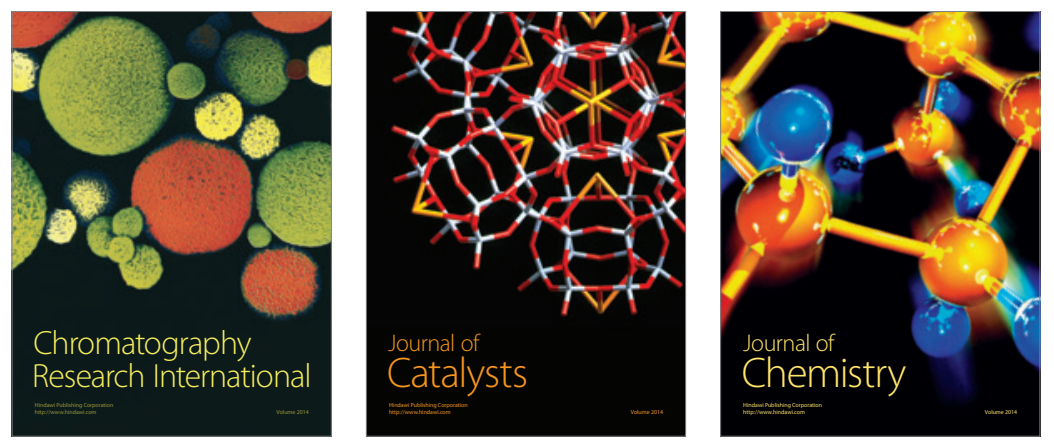
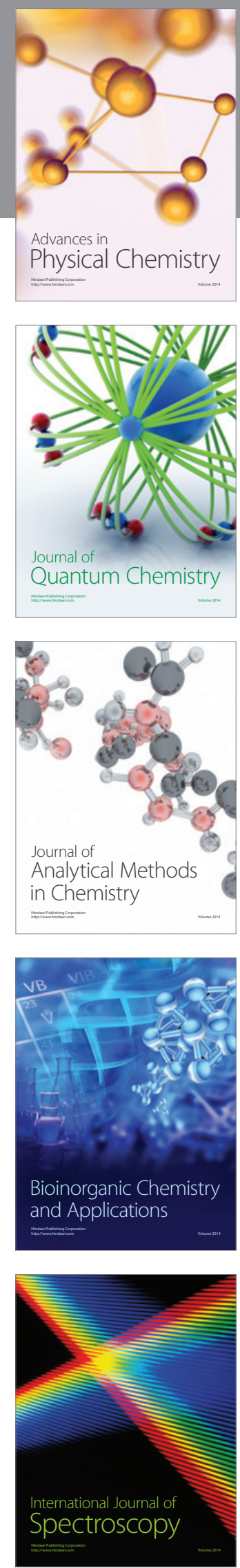